\title{
Manejo quirúrgico laparoscópico de perforación de ciego por palillo de madera
}

\section{Laparoscopic surgical management of cecum perforation by toothpick}

\author{
José Roberto Ramírez-Nava, ${ }^{*}$ Adolfo Cuendis-Velázquez, ${ }^{\ddagger}$ \\ Erick Burgos-Sosa, ${ }^{\S}$ Eduardo Jordan-García*
}

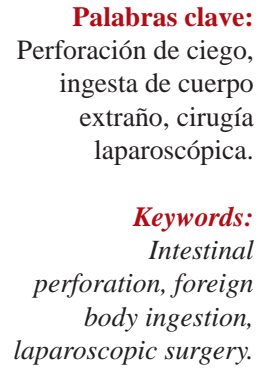

Palabras clave: Perforación de ciego, ingesta de cuerpo extraño, cirugía laparoscópica.

Keywords: Intestinal perforation, foreign body ingestion, laparoscopic surgery.

* Residente de

Cirugía General.

₹ Médico adscrito

al Servicio de

Cirugía General.

$\S$ Médico interno

de pregrado.

Servicio de Cirugía General del Hospital General "Dr. Manuel Gea González”.

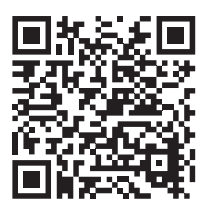

\section{RESUMEN}

Introducción: La presentación clínica de la perforación intestinal secundaria a la ingesta de cuerpos extraños es inespecífica, ya que a menudo el paciente no recuerda la ingesta de éste, y los estudios de imagen resultan negativos, lo que hace difícil el diagnóstico. Material y métodos: Presentamos un caso clínico de perforación de ciego secundario a la ingesta de un palillo de madera, con presentación clínica de abdomen agudo, su diagnóstico y resolución por cirugía laparoscópica. Conclusiones: La ingesta de palillo de dientes puede ser involuntaria durante las comidas. El diagnóstico de perforación gastrointestinal por cuerpos extraños es inespecífico y puede presentarse como una clínica de apendicitis.

\section{ABSTRACT}

Introduction: The clinical presentation of the intestinal perforation, secondary to the intake of foreign bodies usually is inespecific, since the patient often does not remember the intake of this, and the imaging studies are negative, that makes difficult the diagnostic. Material and methods: We present a clinical case of ciego perforation due to intake of toothpick, with clinical presentation of acute abdomen, their diagnostic and their resolution by surgical laparoscopy. Conclusions: The intake of toothpick can be involuntary during meals. The diagnostic of gastrointestinal perforation by foreign bodies is inespecific and can mimic other clinical presentation as appendicitis.

\section{INTRODUCCIÓN}

L a ingesta de cuerpos extraños que alcanzan el estómago pasan de manera inadvertida a través del tracto gastrointestinal, esta situación en ocasiones condiciona la presencia de complicaciones que requerirán una resolución quirúrgica relativamente común. ${ }^{1}$ Se han reportado casos de complicaciones en hasta 35\% con objetos punzantes ingeridos. Las áreas más comunes de perforación es donde existe angulación (esófago superior e inferior, píloro y válvula ileocecal), con mayor frecuencia en íleon (54\%), apéndice y colon (39\%). Los objetos extraños son los huesos de pescado en $55 \%$, seguido de huesos de pollo, con un predominio de afección por el palillo de madera en duodeno, alcanzando una mortalidad de $18 \% .^{1-3}$
Para el diagnóstico de una perforación de víscera hueca por objeto extraño es necesario realizar una correcta anamnesis, puesto que sólo $12 \%$ de los pacientes llega a recordar la ingesta del mismo. ${ }^{2,3}$ De forma inicial, se prefiere la toma de radiografía de tórax y abdomen, ya que se puede sospechar la localización, el tamaño, la forma y el número de los objetos ingeridos. En los pacientes en los que ha sido incapaz la evidencia de cuerpos extraños, es sugerente la realización de una tomografía computarizada, ya que puede identificar de $80-100 \%$ de éstos; y en los demás pacientes que no es posible identificar el objeto extraño, con dolor abdominal agudo se requiere de una exploración quirúrgica. ${ }^{3-8}$

En este reporte de caso presentamos a una paciente con cuadro de abdomen agudo secun-

Citar como: Ramírez-Nava JR, Cuendis-Velázquez A, Burgos-Sosa E, Jordan-García E. Manejo quirúrgico laparoscópico de perforación de ciego por palillo de madera. Cir Gen. 2020; 42 (3): 219-222. https://dx.doi.org/10.35366/99963 
dario a perforación de ciego por objeto extraño, con una clínica muy particular, diagnosticado por cirugía laparoscópica.

\section{CASO CLÍNICO}

Este es el caso de un paciente femenino de 49 años de edad con antecedentes personales importantes de apendicectomía abierta en la infancia, histerectomía abdominal secundaria a miomatosis uterina y ooforectomía sin recordar la causa, aproximadamente hace 25 años.

Inició su cuadro clínico con presencia de dolor localizado en epigastrio y posterior irradiado a fosa iliaca derecha con duración de 20 horas, sin otras irradiaciones, tipo punzante, de intensidad 10/10, que aumenta con la movilidad y sin mejoría aparente, acompañado de escalofríos y alza térmica no cuantificada, otros síntomas negados, sin tratamiento médico previo.

A la exploración física se encontró con dolor en el abdomen a la palpación profunda en fosa iliaca derecha; punto de McBurney; signo de obturador, psoas y signo de Von Blumberg posi-
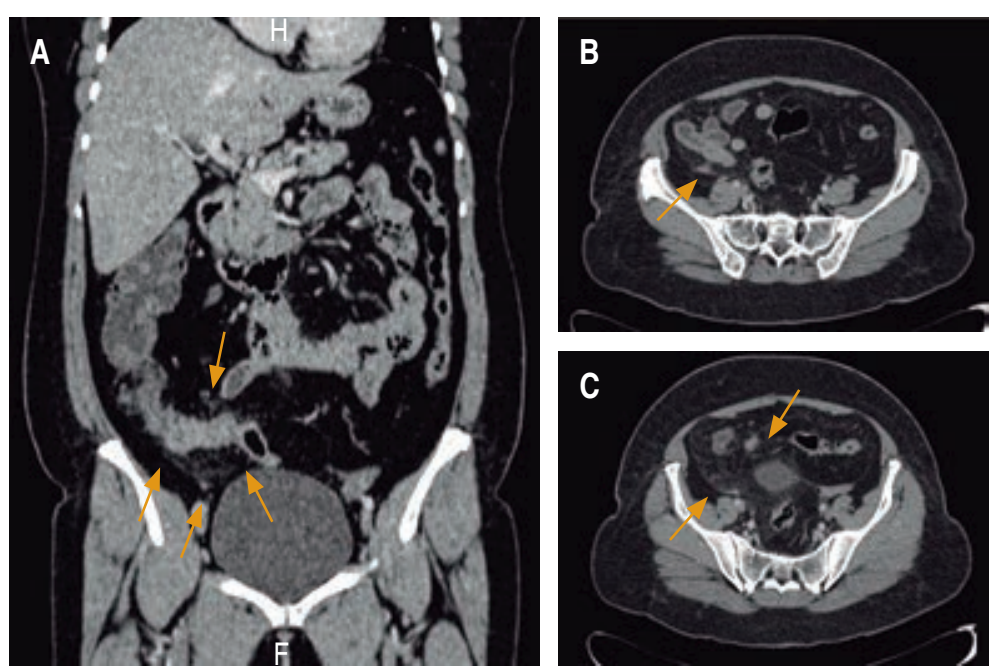

Figura 1: A) Corte coronal, en el que se visualiza realce periférico de la mucosa de asas de intestino delgado y los cambios por estriación de la grasa adyacente. B) Tomografía de abdomen corte axial, posterior a la administración de material de contraste, se identifica aumento en la densidad de la grasa mesentérica en topografía de la fosa iliaca derecha adyacente al ciego, así como realce periférico de la mucosa del mismo, lo cual se asocia a múltiples imágenes de aspecto nodular correspondientes con ganglios de caracteristicas inflamatorias. C) Cambios inflamatorios de la grasa mesentérica que se extiende hacia el hueso pélvico. tivos; puntos ureterales derechos superior y medio; Giordano derecho positivo. Se solicitaron exámenes de laboratorio (biometría hemática, química sanguínea y reactantes de fase aguda) que mostraron como únicos hallazgos importantes leucocitosis a expensas de neutrofilia en $94 \%$ y proteína C reactiva en $4.3 \mathrm{mg} / \mathrm{dl}$. Como estudios complementarios del cuadro de dolor abdominal se realizó tomografía computarizada de abdomen simple y con contraste intravenoso (Figura 1), sin identificación de apéndice cecal y con cambios inflamatorios en grasa pericolónica del lado derecho, reportado por el Servicio de Radiología.

Por lo anterior, se decidió su ingreso para continuar protocolo de estudio, posterior a seis horas y sin evidencia clínica de mejoría se propuso tratamiento quirúrgico y se determinó realizar abordaje laparoscópico inicialmente. Durante la cirugía en la laparoscopia diagnóstica se observó escaso líquido turbio en corredera parietocólica derecha y adherencia laxa de epiplón a pared de fosa iliaca izquierda. Se realizó disección de adherencias laxas de epiplón a ciego y se observó objeto extraño puntiagudo saliente en cara anterior del mismo con fuga de material intestinal. Se retiró el cuerpo extraño con pinzas grasper y se identificó palillo de madera; se realizó cierre primario de perforación con polipropileno 3-0 con nudo extracorpóreo en cruz y se colocó drenaje a corredera parietocólica derecha y hueco pélvico tipo Jackson-Pratt (Figura 2).

Durante el postquirúrgico la paciente evolucionó de manera favorable, mantuvo signos vitales normales, toleró la dieta vía oral, con gasto mínimo por el drenaje de aspecto serohemático, con manejo antimicrobiano con ceftriaxona 1 gramo intravenoso cada 12 horas y metronidazol $500 \mathrm{mg}$ intravenoso cada ocho horas, logrando buena evolución, por lo que fue egresada a las 48 horas después de la cirugía.

\section{DISCUSIÓN}

La ingesta de cuerpos extraños puede ocasionar una perforación intestinal a cualquier nivel. El píloro, el ángulo de Treitz, el íleon terminal y la unión rectosigmoidea son los 

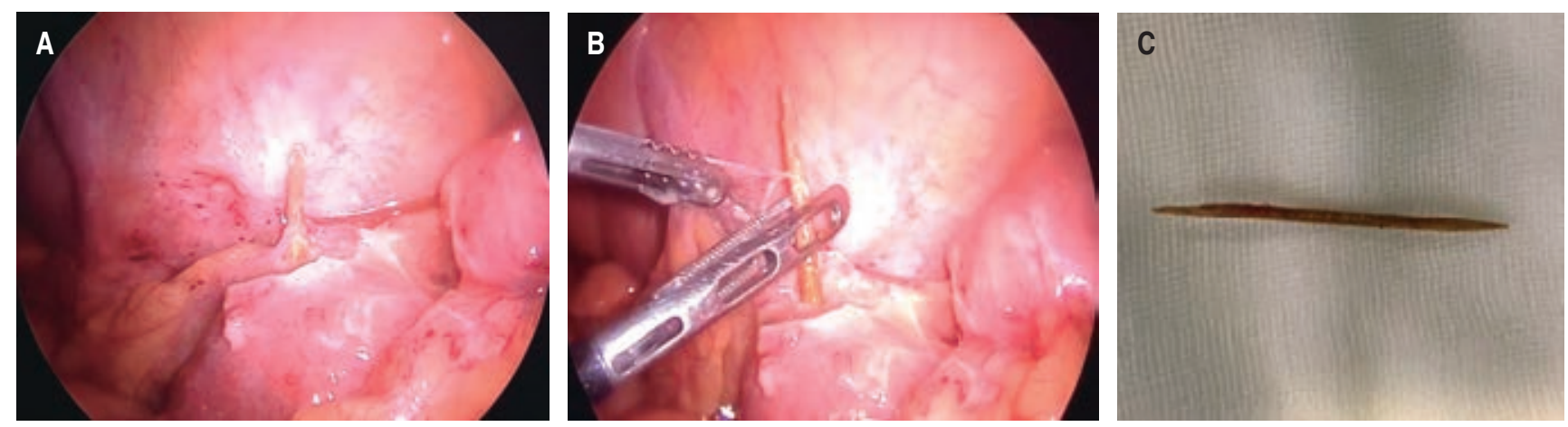

Figura 2: A) Se observa plastrón inflamatorio y palillo de madera que perfora ciego. B) Extracción de objeto extraño con pinzas laparoscópicas. C) Extracción exitosa del palillo de madera.

segmentos más afectados debido a su gran angulación, siendo en nuestra paciente a nivel de ciego. La clínica de la perforación intestinal puede semejar otras condiciones de emergencia como apendicitis aguda, diverticulitis aguda y úlcera péptica perforada, y en algunos casos asociarse a estas patologías, inclusive a algún tumor, pero en una paciente con antecedente de apendicectomía y datos clínicos muy sugerentes de apendicitis hace difícil la sospecha de ésta, ya que esta perforación puede incluso ser asintomática. ${ }^{5-12}$

En nuestra paciente, a pesar de un interrogatorio exhaustivo, no se pudo identificar algún factor de riesgo como alcoholismo, enfermedades psiquiátricas, uso de dentaduras (disminuyen la sensibilidad táctil y del paladar) u otros, como la disminución de la agudeza visual. Aunque probablemente los mismos antecedentes quirúrgicos (ooforectomía, apendicectomía e histerectomía) condicionaron la formación de adherencias, esto no se relaciona con la perforación y la subsecuente perforación por el mondadientes. ${ }^{9-13}$

En el estudio retrospectivo de Ngan y colaboradores, con 358 pacientes que ingirieron hueso de pescado, la radiografía de abdomen obtuvo sólo una sensibilidad de $32 \%$, lo que lo convierte en un estudio con poca sensibilidad, debido a que el objeto extraño es pequeño y tiene poca radioopacidad. Sin la identificación de este patrón, ni la presencia de aire libre en la cavidad de nuestro caso, se realizó una tomografía computarizada de abdomen, ya que puede identificar el cuerpo extraño hasta en 80-100\% de los casos, lo cual convierte a este estudio en el más valioso para el diagnóstico de una perforación intestinal. ${ }^{12-15}$

Por último, se realizó procedimiento laparoscópico como método diagnóstico para identificar la etiología del dolor y de la respuesta inflamatoria sistémica. En algunos casos el abordaje abierto y el laparoscópico en perforación intestinal muestran resultados similares; sin embargo, la laparoscopia ha demostrado un menor íleo paralítico, rápido retorno de la función intestinal, menor dolor y una menor estancia hospitalaria, por lo que se considera como el método de elección en el tratamiento. ${ }^{14-16}$

\section{CONCLUSIONES}

La ingesta de cuerpos extraños puntiagudos que desencadenan una perforación intestinal es de origen accidental en la mayoría de los casos, por lo general tienen presentación clínica atípica y hallazgos radiológicos inespecíficos, por lo que se deben descartar múltiples diagnósticos diferenciales, siendo un reto diagnóstico.

En casos de abdomen agudo la cirugía laparoscópica es una útil herramienta diagnóstica y terapéutica, no sólo al ofrecer el diagnóstico definitivo, sino que dependiendo de los hallazgos durante el procedimiento, en un centro con el equipo y la experiencia adecuada, se puede ofrecer como una opción terapéutica segura y con resultados satisfactorios para el paciente, con una menor estancia hospitalaria, menor presencia de complicaciones postoperatorias y retorno a las actividades normales con mayor rapidez. 


\section{REFERENCIAS}

1. Sarwa P, Dahiya RS, Nityasha, Anand S, Chandrabhan, Gogna S, et al. A curious case of foreign body induced jejunal obstruction and perforation. Int J Surg Case Rep. 2014; 5: 617-619.

2. Kaoutzanis C, Saghir W, Hamade A, Garrett W. Accidental ingestion of a cocktail stick. BMJ Case Reports. 2010; 2010.

3. Sevillano C, Morana MN, Estevez S. Visual involvement in foreign-body intestinal perforations. Arch Soc Esp Oftalmol. 2016; 91: 20-22.

4. Yadav AK, Malla G, Deo KB, Giri S, Bhattarai BM, Adhikary S. Jejunal perforation due to ingested buffalo bone mimicking acute appendicitis. BMC Research Notes. 2016; 9: 321.

5. Sarici IS, Topuz O, Sevim Y, Sarigoz T, Ertan T, Karabiyik O, et al. Endoscopic management of colonic perforation due to ingestion of a wooden toothpick. Am J Case Rep. 2017; 18: 72-75.

6. Perko ZBK, Pogorelic Z, Druzijanic N, Srsen D, Kraljevic D, Juricic J, et al. Acute appendicitis and ileal perforation with a tootpick treated by laparoscopy. Coll Antropol. 2008; 32: 307-309.

7. Chia DK, Wijaya R, Wong A, Tan SM. Laparoscopic management of complicated foreign body ingestion: a case series. Int Surg. 2015; 100: 849-853.

8. Kuzmich S, Burke CJ, Harvey CJ, Kuzmich T, Andrews $\mathrm{J}$, Reading $\mathrm{N}$, et al. Perforation of gastrointestinal tract by poorly conspicuous ingested foreign bodies: radiological diagnosis. Br J Radiol. 2015; 88: 20150086

9. Rodriguez HJ, Codina CA, Olivet PF, Pont VJ, Girones VJ, Roig GJ, Blanco DJ. Perforaciones intestinales causadas por cuerpos extraños. Cirugía Española. 2001;69:504-6.

10. Costa AC, Rainho R, Gouveia A. Codfish may cause acute abdomen. Int J Surg Case Rep. 2013; 4: 969971.

11. Terrace JD, Samuel J, Robertson JH, Wilson RG, Anderson DN. Chicken or the leg: sigmoid colon perforation by ingested poultry fibula proximal to an occult malignancy. Int J Surg Case Rep. 2013; 4: 945947.

12. Akhtar SMN, Gardiner KR, Irwin ST. Bowel perforation caused by swallowed chiken bones-- a case series. Ulster Med J. 2006; 76: 37-38.

13. Pan CC, Wang CP, Huang JJ, Chen WK, Yang HR. Intestinal perforation after the incidental ingestion of a fishhook. J Emerg Med. 2010; 38: e45-48.

14. Ngan JH, Fok PJ, Lai EC, Branicki FJ, Wong J. A prospective study on fish bone ingestion. Experience of 358 patients. Annals of Surgery. 1990; 211: 459-462.

15. Nicolodi GC, Trippia CR, Caboclo MF, de Castro FG, Miller WP, de Lima RR, et al. Intestinal perforation by an ingested foreign body. Radiol Bras. 2016; 49: 295-299.

16. De Bakker J, Bruin S. Successful laparoscopic repair of a large traumatic sigmoid perforation. J Surg Case Rep. 2012; 2012: 3.

Conflicto de intereses: Los autores declaran no tener conflicto de intereses.

\section{Correspondencia:}

Dr. José Roberto Ramírez Nava

E-mail: dr.joserobertonava@gmail.com 